\title{
9 Wie Industrieproduktion nachhaltig gestaltet werden kann
}

Stefan Wolf, Max Michael Jordan, Inessa Seifert, Marco Evertz, Roman Korzynietz

Auch wenn bereits Fortschritte hin zu einer nachhaltigeren Industrieproduktion erzielt wurden, so ist der Status quo noch weit von wirklicher Nachhaltigkeit entfernt. Um einen nachhaltigen Gleichgewichtszustand zu erreichen, muss die Emission von Treibhausgasen fossilen Ursprungs in die Atmosphäre gänzlich eingestellt werden. Dieses ist verbunden mit einer tiefgreifenden Transformation der Industrie.

Die industrielle Produktion von Gütern aller Art trägt in erheblichem Maße zur übermäßigen Ausbeutung natürlicher Ressourcen bei. Umweltauswirkungen aus der Extraktion und Weiterverarbeitung von Rohstoffen, aus der Veränderung von Lebensräumen und der Versiegelung von Flächen sowie aus der Emission von Lärm, Schadstoffen, Staub und Treibhausgasen spiegeln sich zumeist nicht im Preis der Industrieprodukte wider. Die entstehenden Schäden sind externalisiert; sie werden von der Gesellschaft getragen. Immer wieder mahnen spektakuläre Katastrophen vor Umweltzerstörungen durch industrielle Aktivitäten, wie etwa bei den Dammbrüchen im brasilianischen Brumadinho im Jahr 2019 oder im ungarischen Devecser im Jahr 2010. Beide Male überschwemmten Millionen Kubikmeter giftigen Schlamms zuvor intakte Ökosysteme. Gravierender sind aber die schleichenden Prozesse, bei denen die Umweltauswirkungen ihr schädliches Potenzial zeitlich oder örtlich entkoppelt entfalten, wie es beim Ausstoß von Treibhausgasen der Fall ist. Gegenwärtig werden rund 30 Prozent der globalen Treibhausgasemissionen von der Industrie verursacht (Intergovernmental Panel on Climate Change, 2014).

Um einen nachhaltigen Gleichgewichtszustand zu erreichen, ist es unabdingbar, die weitere Emission von Treibhausgasen fossilen Ursprungs in die Atmosphäre gänzlich einzustellen. Dies erfordert auch in der Industrie eine tiefgreifende Transformation aller Prozesse. Es handelt sich dabei um keine leicht zu lösende Aufgabe in einem Spannungsfeld, in dem es gilt, Kunden mit preiswerten Produkten zu versorgen, regulatorische Rahmenbedingungen einzuhalten, die Umwelt zu schonen und zugleich Gewinne zu erwirtschaften. All diese Aspekte miteinander zu vereinen, stellt ein komplexes Optimierungsproblem dar, das nicht von der Industrie allein gelöst werden kann. Es gilt, den Nutzen industrieller Produkte mit den Umweltauswirkungen ins Verhältnis zu setzen und zu entscheiden, welche Folgen im Sinne einer nachhaltigen 
Entwicklung in Kauf genommen werden können und welche nicht. Die Moderation dieses Optimierungsprozesses obliegt der Politik, denn sowohl die Vermessung des Nutzens als auch die Definition von Nachhaltigkeitsgrenzen müssen gesellschaftlich verhandelt werden. Im Rahmen dieses Optimierungsprozesses muss wiederum die Politik die neuen Rahmenbedingungen definieren und setzen, an denen sich wirtschaftliches Handeln und industrielle Produktion künftig ausrichten.

Zur Etablierung einer nachhaltigen und zugleich wettbewerbsfähigen Industrieproduktion müssen die folgenden drei Fragen beantwortet werden:

1. Welche Technologien können die Transformation hin zu einer nachhaltigen Industrieproduktion unterstützen?

2. Wie müssen die Rahmenbedingungen für eine industrielle Produktion im Rahmen der Nachhaltigkeitsgrenzen gestaltet sein?

3. Wie können faire Wettbewerbsbedingungen hergestellt und die Abwanderung von Industrieproduktion vermieden werden?

\section{Umweltauswirkungen der Industrieproduktion}

Dass politische Maßnahmen zur Verbesserung der Nachhaltigkeit von Produktionsverfahren und Produkten beitragen können, zeigt der Erfolg des Montreal-Protokolls. In Folge des Verbots von ozonschädigenden Substanzen schrumpfte das Ozonloch über der Antarktis im Vergleich zum Rekordjahr 2006 bis zum Jahr 2017 um rund 25 Prozent. Ebenso ist bei den Schwefeldioxidemissionen der Industrie in Deutschland eine deutliche und stetige Reduktion um fast 90 Prozent von 1990 bis 2017 festzustellen (Umweltbundesamt, 2019b). Die Beobachtung des Waldsterbens in den 1980er-Jahren, maßgeblich hervorgerufen durch sauren Regen aufgrund von Schwefeldioxidemissionen, war Ausgangspunkt für dieses Gegensteuern der Politik. Die Wasserentnahme für die Nutzung in industriellen Prozessen sowie im Bergbau ist in Deutschland seit 1990 um 47 Prozent zurückgegangen. Neben Treibhausgasemissionen ist die Industrie in Deutschland mit einem Anteil von 43 Prozent auch die Hauptquelle für Feinstaubemissionen der Partikelgröße 10 Mikrometer. Nach anfänglich deutlichen Reduktionen ist hier in der letzten Dekade ebenfalls eine Stagnation zu beobachten. Gleiches gilt für die Emissionen gröberen Staubes, bei denen der Anteil der Industrie sogar 52 Prozent beträgt.

Ungeachtet einiger Erfolge stagnieren die Fortschritte bei einigen wichtigen Nachhaltigkeitsparametern. So sind zum Beispiel in Deutschland die Treibhausgasemissionen der Industrie von 284 Millionen Tonnen $\mathrm{CO}_{2}$-Äquivalenten im Referenzjahr 1990 auf 196 Millionen Tonnen $\mathrm{CO}_{2}$-Äquivalente im Jahr 2018 gesunken. Dies entspricht einer Reduktion um 31 Prozent. Bis zum Jahr 2030 sieht der Klimaschutzplan 2050 


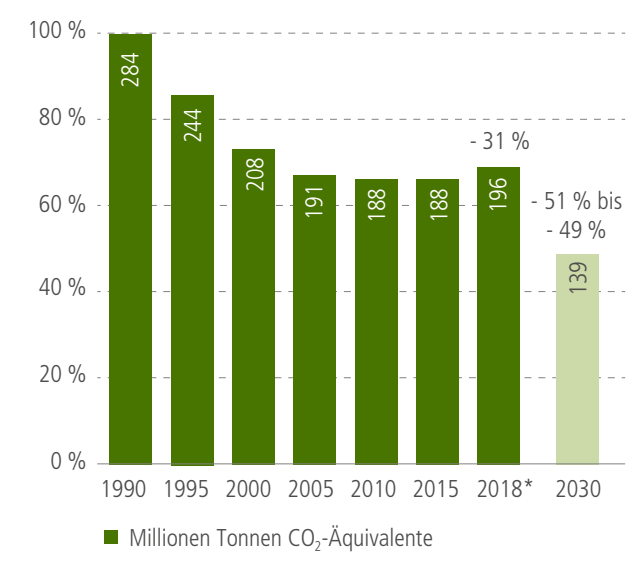

* Vorläufige Daten für 2018

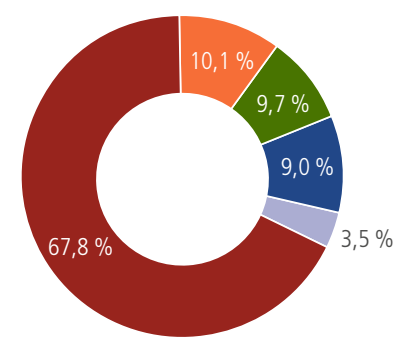

Industriefeuerung ${ }^{* *}$ ohne $\mathrm{CO}_{2}$ - Herstellung von Metall aus verbrannter Biomasse

- Herstellung mineralischer Produkte

- übrige Prozesse und Produktverwendung

- Chemische Industrie

$24,8 \%$ der Industrieemissionen sind prozessbedingt.

** Verbrennungsprozesse, beispielsweise aus der Befeuerung von Drehrohröfen

Abb. 9.1 Treibhausgasemissionen der Industrie in Deutschland. (Eigene Darstellung nach BMU 2019)

der Bundesregierung eine Reduktion um 49 bis 51 Prozent vor. Wie Abb. 9.1 zeigt, stagnieren die Emissionen seit dem Jahr 2005 (BMU, 2019). Die starke Reduktion in den 1990er-Jahren ist zudem wesentlich auf den Niedergang der ostdeutschen Schwerindustrie zurückzuführen. Entsprechend groß ist somit die Gefahr, dass die Klimaziele im Jahr 2030 verfehlt werden, wenn nicht gehandelt wird.

Die größten industriellen Quellen von Treibhausgasen sind Industriefeuerungen im Allgemeinen sowie die Prozesse der Petrochemie, der Metallerzeugung und der übrigen chemischen Industrie. Hier bestehen die größten Hebel für zusätzliche industrieund klimapolitische Ansätze.

Der Klimawandel ist nicht das einzige, wohl aber das zurzeit drängendste Nachhaltigkeitsproblem. Bei einem gleichbleibenden Niveau an Treibhausgasemissionen ist das für Deutschland verbleibende Emissionsbudget zur Einhaltung des globalen 1,5-Grad-Ziels aus dem Pariser Klimaabkommen in weniger als zehn Jahren aufgebraucht (Hausfather, 2018). Einige Studien gehen sogar davon aus, dass dieses Budget bereits heute ausgeschöpft ist. Jede Tonne zusätzlicher Emissionen verstärkt das Risiko, dass unumkehrbare Prozesse wie das Abschmelzen der polaren Eiskappen ausgelöst werden. Damit die Erhaltung des Ökosystems Erde sichergestellt werden kann, muss auch die Industrie ihren Beitrag leisten. 


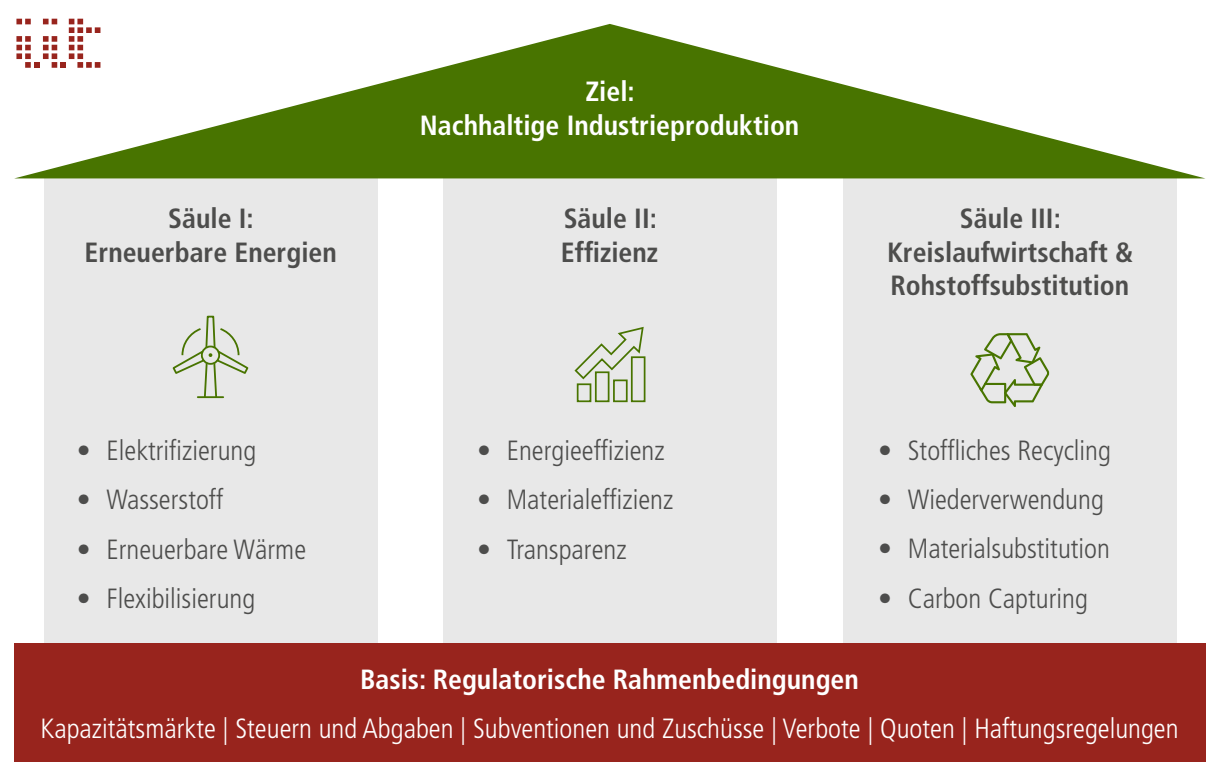

Abb. 9.2 Die drei Säulen einer nachhaltigeren Industrieproduktion. (Eigene Darstellung)

\section{Die drei Säulen einer nachhaltigeren Industrieproduktion}

Die Nachhaltigkeitstransformation der Industrie erfordert ein koordiniertes Vorgehen in den Technologiefeldern "Erneuerbare Energien" und "Effizienzsteigerung" sowie in der "Kreislaufwirtschaft und Rohstoffsubstitution" (siehe Abb. 9.2). Je weniger auf das eine Themenfeld gesetzt wird, desto größer müssen die Anstrengungen in den anderen ausfallen. Für den Erfolg unabdingbar ist die Gestaltung geeigneter regulatorischer Rahmenbedingungen durch die Politik.

Säule 1: Erneuerbare Energien

In der Industrie werden große Mengen an Energie für den Antrieb von Maschinen und die Erzeugung von Wärme benötigt. Rund 25 Prozent des gesamten Endenergieverbrauchs der Europäischen Union verursacht die Industrie (eurostat, 2019). In Deutschland sind es sogar rund 30 Prozent (Umweltbundesamt, 2020). Ein Blick auf die Zusammensetzung des industriellen Energiemixes in Abb. 9.3 verrät, dass vornehmlich fossile Energieträger wie Erdgas, Öl und Kohle genutzt werden. Die Umstellung der Energieversorgung auf erneuerbare Energien ist somit eine der wesentlichen Strategien hin zu einer nachhaltigeren Industrieproduktion.

Die verschiedenen Stromerzeugungstechnologien werden detailliert im Kap. 8 "Herausforderungen einer klimafreundlichen Energieversorgung" des Themenbands 


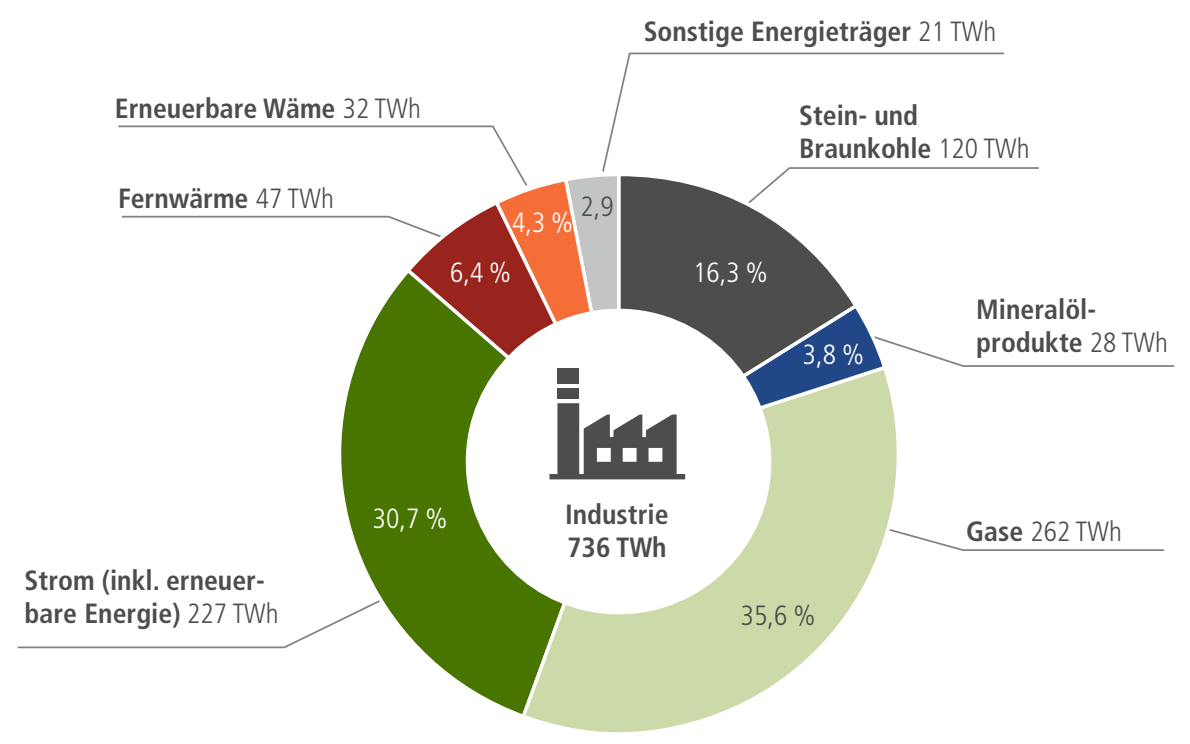

Abb. 9.3 Endenergieverbrauch der Industrie in Deutschland im Jahr 2018. (Eigene Darstellung nach Umweltbundesamt 2020)

beschrieben. An dieser Stelle wird der Fokus auf die Besonderheiten industrieller Produktionsprozesse gelegt. Das betrifft vor allem die Erzeugung von Prozesswärme, die in großen Mengen beispielsweise für die Herstellung von Stahl, Zement, Papier oder chemischen Erzeugnissen benötigt wird. Fast drei Viertel des industriellen Endenergieverbrauchs entfallen auf die Erzeugung von Wärme (Bundesministerium für Wirtschaft und Energie, 2020). Hier spielen erneuerbare Energien bisher kaum eine Rolle. Werden sie doch einmal genutzt, handelt es sich meist um die Verbrennung von biogenen Reststoffen aus der Produktion. In der Industrie besteht ein dringender Bedarf an Lösungen für die Umstellung auf eine nachhaltige und klimaverträgliche Wärmeerzeugung.

Eine Option für eine nachhaltigere Energieversorgung ist die direkte Nutzung erneuerbaren Stroms. Der einfachste und sicherlich wichtigste erste Schritt der direkten Nutzung ist der Wechsel zu einem Ökostromanbieter. Ist dieser Schritt getan, kann die Elektrifizierung von Industrieprozessen zu weiteren Treibhausgaseinsparungen führen. Möglich ist dieses beispielsweise durch einen vermehrten Einsatz von Wärmepumpen und Elektrodenkesseln zur Warmwasser- und Dampferzeugung, von Lichtbogenöfen in der Metallerzeugung oder von UV-Desinfektion und Mikrowellenerwärmung in der Lebensmittelindustrie. Ferner kann Hochtemperaturabwärme mit Dampfprozessen im Organic Rankine Cycle (ORC) oder Kalinaverfahren verstromt werden. 
Prozesse, bei denen eine Elektrifizierung nicht möglich ist, können durch die indirekte Nutzung erneuerbaren Stroms nachhaltiger werden. Hier wird erneuerbare Energie in einen chemischen Energieträger umgewandelt, der dann in Industrieprozessen genutzt werden kann. Dabei wird zunächst erneuerbarer Strom in Elektrolyseuren dazu verwendet, um Wasser in Wasserstoff und Sauerstoff zu spalten. Bei der Umwandlung erneuerbaren Stroms in chemische Energie geht allerdings nutzbare Energie verloren. Neue Elektrolysekonzepte versprechen diesen Nachteil zu verringern. Die Hochtemperatur-Elektrolyse ist beispielsweise in der Lage, unter idealen Bedingungen Wirkungsgrade von mehr als 80 Prozent zu erzielen (o.V., 2019b). Noch ist die Nutzung dieses mit erneuerbaren Energien erzeugten grünen Wasserstoffs vergleichsweise teuer. Das in Planung befindliche „Important Project of Common European Interest (IPCEI) on Hydrogen" und der deutsche Ideenwettbewerb "Reallabore der Energiewende" schaffen die Rahmenbedingungen für die Kommerzialisierung und Skalierung der Wasserstofftechnologie. Zudem können fossile Energieträger durch erneuerbaren Wasserstoff substituiert werden. So wird Wasserstoff beispielsweise im Rahmen des Förderprogramms "IN4climate.NRW" in der Stahlerzeugung als Reduktionsmittel anstelle von Kohlestaub direkt in den Hochofen eingeblasen (o. V., 2019b).

Weiterhin kann erneuerbare Wärme aus Quellen wie Geothermie (Stagge, 2019) und Solarthermie erzeugt werden, deren Potenzial bisher weder in Deutschland noch international in nennenswertem Maß ausgeschöpft ist. Die Verfahren sind technisch weit fortgeschritten. Solarthermische Parabolrinnen- oder Fresnelkollektoren werden zur Erzeugung von Prozessdampf in Regionen mit hoher direkter Sonneneinstrahlung eingesetzt. Dass auch Hochtemperaturprozesse bis zu 1000 Grad Celsius versorgt werden können, wurde zum Beispiel im SOLPART-Projekt demonstriert (o. V., 2019a). Auch bei der in Deutschland überwiegenden diffusen Sonneneinstrahlung können solarthermische Anlagen zur Warmwassererzeugung beispielsweise in der Lebensmittelindustrie genutzt werden. Hemmend wirken sich gegenwärtig noch vergleichsweise hohen Kosten sowie die aufwendigere Anlagenplanung und -installation aus.

Die Einbindung volatiler erneuerbarer Energien in die industrielle Energieversorgung erfordert die Mitwirkung und Flexibilisierung der Nachfrageseite. Lastmanagement ermöglicht die flexible Steuerung von Produktionskapazitäten und damit auch des Energiebedarfs. Damit werden Industrieunternehmen, nach erfolgreicher Präqualifizierung, zu Anbietern von Regelenergie und leisten einen Beitrag zur Stabilisierung des Energiesystems. Bei der Umsetzung ist eine Vielzahl von Faktoren zu berücksichtigen, damit es nicht zur Beeinträchtigung der Produktgüte, zu schnellerem Anlagenverschleiß, zu höheren Produktionskosten oder zu negativen sozialen Auswirkungen führt. 


\section{Säule 2: Effizienz}

Effizienz ist die zweite Säule in der klimaneutralen und nachhaltigen Transformation der Industrie. Denn Ressourcen, die nicht extrahiert und verbraucht werden, entfalten keine negativen Umweltauswirkungen. Die Effizienz versteht sich dabei als Verhältnis des erreichten Nutzens zum Aufwand. So hat die Emission von Treibhausgasen bezogen auf die Bruttowertschöpfung der Industrie seit 1995 um 19 Prozent abgenommen. Die Gründe dafür sind die gestiegene Effizienz der Produktionsprozesse sowie auch die vermehrte Nutzung erneuerbarer Energien.

Die Steigerung der Energieeffizienz ist ein sehr wirksames Instrument zur Verbesserung der Nachhaltigkeit der industriellen Produktion, besonders gilt dies für die energieintensive Prozessindustrie. Hier sind bereits energetische Optimierungsverfahren wie die Pinch-Analyse etabliert. Mit bereits verfügbaren Technologien können allein in der energieintensiven Prozessindustrie in Deutschland bis zu 18 Prozent des Energiebedarfs eingespart werden (Brunke, 2017). Große Potenziale zur Effizienzsteigerung bestehen vor allem in der Wärmerückgewinnung. Zur systematischen Optimierung des Energiebedarfs können Energiemanagementsysteme (EnMS) eingesetzt werden. Die Einführung eines EnMS führt zu Einsparungen von 2,5 bis 15 Prozent (Matteini et al., 2018). Unterstützt durch Sensorik, Kommunikationstechnologie, intelligente Datenverarbeitung und Künstliche Intelligenz (KI) können EnMS einen wesentlichen Beitrag zur flexiblen und effizienten Energienutzung in Industriebetrieben leisten (Zinke, 2019).

Ein nicht zu unterschätzender Beitrag zur Verbesserung der Nachhaltigkeit in der Industrieproduktion ist die Steigerung der Materialeffizienz. Ein geringerer Materialeinsatz hat unmittelbar einen reduzierten Bedarf an Rohstoffen zur Folge und damit auch einen geringeren Bedarf an deren Extraktion und Verarbeitung. Auf diese Weise entfallen vielfach gravierende Eingriffe in natürliche Ökosysteme.

Die wachsende Verfügbarkeit von Daten und Rechenleistung eröffnet ebenfalls neue Wege zur Steigerung der Materialeffizienz. Über cloudbasierte Internet-ofThings(IOT)- und Industrie-4.0-Plattformen erhalten auch kleine und mittelständische Unternehmen Zugang zu KI-basierten Services. Als eine von 16 Plattformen im Innovationswettbewerb „Künstliche Intelligenz als Treiber für volkswirtschaftlich relevante Ökosysteme" verspricht IIP-Ecosphere die Steigerung der Produktivität, Flexibilität, Robustheit und Effizienz von Produktionsprozessen. Die durch Digitalisierung erschließbaren Materialeinsparpotenziale sind in der deutschen Industrie längst nicht vollständig ausgeschöpft. So können mit Digitalisierungstechnologien und der Substitution umweltbelastender Rohstoffe durch nachhaltige Materialien 3 bis 4 Prozent des gesamten Materialverbrauchs eingespart werden. Der Wert dieser Einsparungen wird auf knapp zwei Milliarden Euro geschätzt (Neligan and Schmitz, 2017). 
In globalisierten Wirtschaftsbeziehungen ist Transparenz eine wichtige Voraussetzung dafür, dass hohe Effizienz und Nachhaltigkeit entlang der gesamten Wertschöpfungskette erreicht werden können. Die Anwendung der Normen DIN EN ISO 50001 Energiemanagementsysteme, DIN EN ISO 14001 Umweltmanagementsysteme und die Zertifizierung nach dem Eco-Management and Audit Scheme (EMAS) bieten einen strukturierten Ansatz zur Abbildung von Nachhaltigkeitsbestrebungen in Unternehmensprozessen. Mit diesen Systemen werden unter anderem auch Kennzahlen erhoben, an denen Unternehmen ihre Fortschritte hin zu einer nachhaltigeren Produktion messen können.

\section{Säule 3: Kreislaufwirtschaft und Rohstoffsubstitution}

Ziel der Kreislaufwirtschaft ist es, Materialkreisläufe zu schließen, in denen nicht mehr benötigte Produkte wiederverwendet oder recycelt werden. Das geschieht beispielsweise in der Herstellung und dem stofflichen Recycling von Produkten der Hochleistungselektronik wie Smartphones, Laptops, Monitoren oder Fernsehern. Darin befinden sich häufig signifikante Mengen an Metallen wie Gold oder Seltenen Erden, zu denen insgesamt 17 chemische Elemente zählen. Seltenerdmetalle werden häufig unter ökologisch und ethisch kritischen Bedingungen in Schwellen- und Entwicklungsländern, meist in Afrika, gefördert. Allein die nicht mehr genutzten Smartphones in Deutschland, von ihren Besitzern in Schränken und Schubladen gehortet, haben einen Goldwert von rund 130 Millionen Euro (bei 3 Tonnen Gold) (o.V., 2020b). Derzeit gibt es keine europäische Regulierung zum Recycling dieser Produktklasse. Erste Ideen werden aber bereits diskutiert. Es könnte ein komplementäres System aufgebaut werden, wie einst bei den Blei-Akkumulatoren, die als Starterbatterie in Pkw verwendet werden. Wie Abb. 9.4 verdeutlicht, ist die Recyclingrate bei BleiAkkumulatoren im Vergleich zu anderen Produktklassen überragend gut.

Die Batterien sind bei der Etablierung einer Kreislaufwirtschaft in zweierlei Hinsicht interessant. Sie sind einerseits Enabler einer nachhaltigeren Energieversorgung. Gleichzeitig sind sie aufgrund der material- und energieintensiven Herstellung Gegenstand von Nachhaltigkeitsdiskussionen. Die europäische Richtlinie zum Batteriezellenrecycling (2006/66/EG) aus dem Jahr 2006 befindet sich derzeit in der Überarbeitung, da damals die hohe Entwicklungsdynamik in der Batterietechnologie noch nicht abzusehen war. Bis zum Jahr 2030 wird ein Marktwachstum von über 600 Prozent erwartet (Michaelis et al., 2018). Um den Rohstoffbedarf in Grenzen zu halten, werden effiziente Recyclingverfahren benötigt. In einer modernen Fahrzeugbatterie mit 50 Kilowattstunden Kapazität stecken zum Beispiel 100 Kilogramm Kohlenstoff, 32 Kilogramm Nickel, 11 Kilogramm Kobalt und 6 Kilogramm Lithium (Rudschies, 2019). Im gegenwärtig genutzten pyrometallurgischen Recyclingverfahren können Metalle wie Lithium, Eisen und Aluminium sowie der Kohlenstoff und der Elektrolyt der Batterie nicht zurückgewonnen werden. Hydrometallurgische Recyclingverfahren 


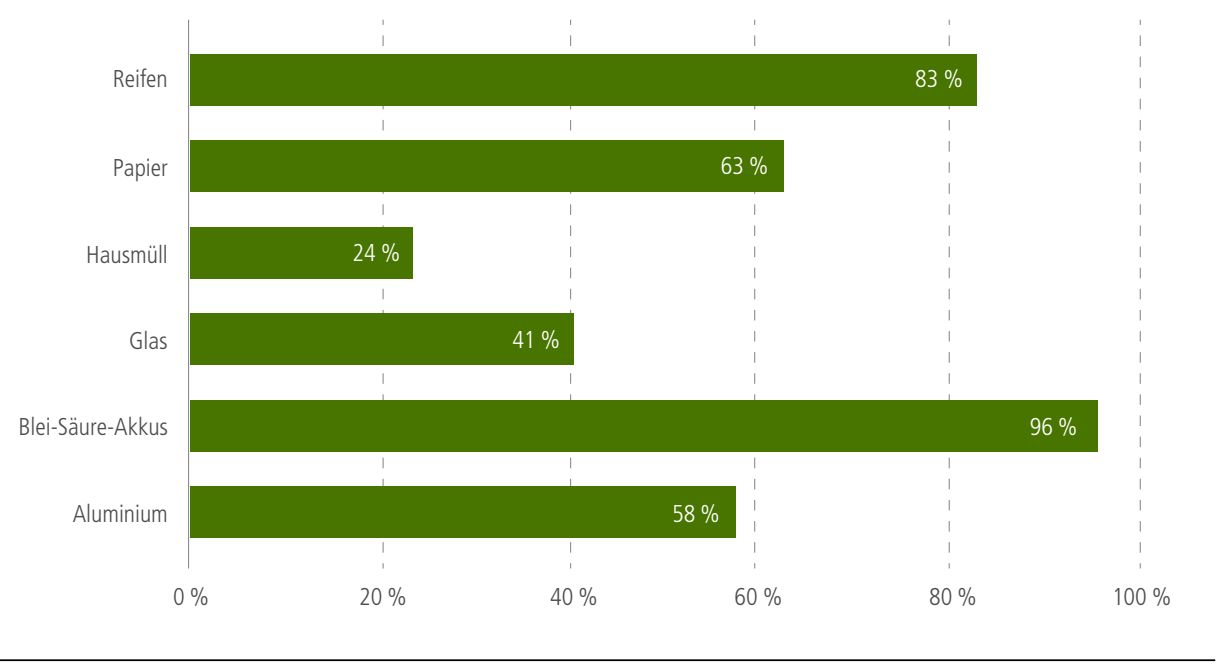

Abb. 9.4 Recyclingraten für unterschiedliche Materialien in den USA. (Aus Argonne National Laboratory Chicago, eigene Darstellung nach Prengaman and Mirza, 2017)

erreichen hingegen Recyclingquoten von bis zu 90 Prozent und können gleichzeitig den Energiebedarf und die $\mathrm{CO}_{2}$-Emissionen deutlich verringern (Diekmann et al., 2018). Dieses Verfahren ist derzeit allerdings aufgrund geringer Rückläufe alter Lithium-lonen-Batteriezellen, hoher Kosten und komplexer Prozessabläufe kaum wirtschaftlich umsetzbar.

Bevor jedoch überhaupt ein stoffliches Recycling in Erwägung gezogen wird, sollten Möglichkeiten zur Wiederverwendung ausgeschöpft werden, um ein Produkt einer erneuten Nutzung zuzuführen. Ein Beispiel dafür sind Pfandflaschen, die nach der Nutzung wieder gesammelt und erneut befüllt werden. Bei Batterien von Elektrofahrzeugen ist eine Wiederverwendung in einem anderen Nutzungskontext möglich. Der Großteil der Fahrzeughersteller garantiert nach acht Jahren bzw. 160.000 gefahrenen Kilometern eine Restkapazität von mindestens 70 Prozent (Harloff, 2019). Danach können Batterien in Anwendungen mit geringerer Batteriebelastung weiterverwendet werden. Beispielsweise setzt BMW Batteriezellen aus der Modellreihe des i3 in der Speicherfarm im Werk Leipzig als stationäre Stromspeicher ein (Müller, 2017).

Ein weiterer Weg zu nachhaltigeren Produktionsverfahren und Produkten ist die Materialsubstitution fossiler Rohstoffe durch biobasierte. So können Chemikalien, Kunst- und Brennstoffe unter Nutzung nachwachsender Rohstoffe auch biotechnologisch in Bioraffinerien hergestellt werden. Besonders ergiebig ist eine Umstellung von einer petrochemischen hin zu einer biobasierten Produktion in der Spezial- und Feinchemie. Lag der Anteil biobasierter Fein- und Spezialchemikalien im Jahr 2005 
auf dem US-amerikanischen Markt noch bei etwa 4 Prozent, so wird erwartet, dass er bis zum Jahr 2025 auf 44 bis 50 Prozent ansteigen wird (Philp, Ritchie and Allan, 2013). Bioraffinerien nutzen meist Kohlenhydrate aus landwirtschaftlich angebauten Getreiden oder Feldfrüchten als Kohlenstoffquelle. Problematisch sind der hohe Flächenbedarf und der Umstand, dass diese Flächen der Produktion von Nahrungsmitteln entzogen werden. Damit verbundene Verdrängungseffekte bedrohen zudem tierische und pflanzliche Lebensräume. Neuere biotechnologische Verfahren nutzen daher Lignocellulose aus Agrarreststoffen.

Schließlich sind Produktionsverfahren eine große Herausforderung, die prozessbedingt Treibhausgase emittieren. Dies ist beispielsweise in der Zementherstellung der Fall, die allein für rund 8 Prozent der globalen Treibhausgasemissionen verantwortlich ist. Nicht vermeidbare prozessbedingte Emissionen können durch Carbon Capture and Utilization (CCU) kompensiert werden. Dabei wird $\mathrm{CO}_{2}$ aus der Luft oder aus Rauchgasen abgeschieden und, meist in Verbindung mit Wasserstoff, für die Produktion kohlenstoffhaltiger Produkte genutzt. Die technologische Basis für die Bereitstellung des Wasserstoffs liefern Power-to-X-Verfahren, bei denen Stromüberschüsse aus erneuerbaren Energiequellen für die elektrolytische Produktion von Wasserstoff verwendet werden. In nachgelagerten oder teils auch kombinierten (bio) katalytischen Prozessen erfolgt dann die Reaktion des Wasserstoffs mit $\mathrm{CO}_{2}$ zu Kohlenwasserstoffen, die entweder direkt verwendet oder als Intermediate weiter zu den spezifisch gewünschten Chemikalien umgesetzt werden. Ein Beispiel hierfür ist das Rheticus-II-Projekt von Siemens und Evonik. In Marl errichten die Unternehmen eine Demonstrationsanlage, bei der ein $\mathrm{CO}_{2}$-Elektrolyseur mit einem Bioreaktor kombiniert wird, um in Industrieprozessen anfallendes $\mathrm{CO}_{2}$ für die Produktion von Butanol und Hexanol zu nutzen (Ahrens, 2019). Auf diese Weise kann mit der Herstellung einer Tonne Butanol die Emission von drei Tonnen $\mathrm{CO}_{2}$ vermieden werden (Weinhold, 2019). Prinzipiell können in derartigen Verfahren erzeugte Kohlenwasserstoffe nicht nur stofflich, sondern auch energetisch genutzt werden. Diese chemischen Energieträger können in großen Mengen und über lange Zeiträume gespeichert werden. Bedingt durch die unvermeidbaren Energieverluste bei der Stromkonversion werden synthetische Energieträger jedoch im Mittel teurer sein als regenerativ erzeugter Strom.

\section{Regulatorische Maßnahmen bilden die Erfolgsgrundlage}

Die Transformation der Industrie zu einer nachhaltigen Produktionsweise benötigt Regeln, die eine adäquate Abwägung von Ressourceninanspruchnahme und Umweltfolgen überhaupt erst ermöglichen. Diese Regeln müssen die Knappheit der natürlichen Ressourcen widerspiegeln. Das setzt einen gesellschaftlichen Abstimmungs- 
prozess voraus, in dem ausgehandelt werden muss, welche Umweltauswirkungen im Zuge der industriellen Aktivität in Kauf genommen werden.

Ein zentrales Problem dabei ist die optimale Allokation der begrenzten Ressourcen. Eine marktorientierte Lösung können Kapazitätsmärkte bieten. Bei diesem Vorgehen werden die Obergrenzen der Ressourceninanspruchnahme vorgegeben und die Anteile meistbietend versteigert. Unzumutbaren Härten kann durch Zuteilung von Ressourcenbudgets begegnet werden. Der Vorteil solcher Kapazitätsmärkte ist die unbedingte Einhaltung der vereinbarten Ressourcenobergrenze.

Beispiel für dieses Prinzip ist das europäische Emissionshandelssystem, in dem 11.000 Anlagen und rund 40 Prozent der gesamten $\mathrm{CO}_{2}$-Emissionen der 31 teilnehmenden Länder erfasst sind (Umweltbundesamt, 2019a). Die Zuteilung von Emissionsrechten erfolgt nach einem komplexen Verfahren, das die besonderen Erfordernisse der einzelnen Marktteilnehmer würdigt. Das verfügbare Emissionsbudget wird jährlich um 1,74 Prozent und ab 2021 um 2,2 Prozent reduziert (European Commission, 2015), was voraussichtlich einen steigenden $\mathrm{CO}_{2}$-Preis zur Folge hat. Das deutsche Brennstoffemissionshandelsgesetz, auch $\mathrm{CO}_{2}$-Steuer genannt, ist eine stark vereinfachte Version eines Kapazitätsmarkts, bei dem zunächst keine freie Preisbildung zugelassen ist.

Weiterhin können Steuern und Abgaben eine Lenkungsfunktion zur Einhaltung von Nachhaltigkeitsanforderungen entfalten. Die Einhaltung von Ressourcenobergrenzen kann allerdings nur durch ein fortlaufendes Monitoring mit ständiger Nachsteuerung sichergestellt werden. Damit sind nicht nur die Stellgrößen, sondern das Lenkungssystem aus Steuern und Abgaben selbst Gegenstand politischer Debatten. Das birgt die Gefahr, Nachhaltigkeitsansprüche kurzfristigen Vorteilen durch nicht nachhaltiges Handeln zu opfern. Der Vorteil liegt indes darin, dass auf Steuern und Abgaben basierende Lenkungssysteme im Vergleich zu Kapazitätsmärkten leichter zu entwickeln und umzusetzen sind. Ein Beispiel für die steuerliche Begünstigung nachhaltigen Handelns sind die Energiesteuer nach Energiesteuergesetz (EnergieStG) oder die Stromsteuer nach Stromsteuergesetz (StromStG).

Subventionen und Zuschüsse sind ein Instrument der positiven Anreizsetzung. Damit kompensiert der Staat Wettbewerbsverzerrungen oder andere finanzielle Nachteile, die durch nachhaltiges Verhalten entstehen können. Beispielsweise können stromintensive Industrien eine Strompreiskompensation erhalten, um indirekte Belastungen durch den europäischen Emissionshandel auszugleichen. Damit bleiben sowohl die Steuerungswirkung des Emissionshandels als auch die Wettbewerbsfähigkeit der stromintensiven Industrie erhalten.

Verbote können gesellschaftlich nicht akzeptierte Verhaltensweisen unterbinden. Allerdings: Verbote müssen kontrolliert und Fehlverhalten mit Strafen belegt wer- 
den. Strafen entfalten zum einen wirtschaftliche Anreize, Verbote einzuhalten, zum anderen ist der Erhalt einer Strafe aber auch mit einer gewissen Stigmatisierung verbunden. Dementsprechend üben Strafen auch einen gesellschaftlichen Druck aus, Regeln einzuhalten. Ein Beispiel für eine Verbotsregelung ist das Verbot ozonabbauender Substanzen gemäß der europäischen Umsetzung des Montreal-Protokolls (EG 1005/2009). Der Übergang zu einem Verbot kann auch durch ein Quotenmodell realisiert werden, wie es die Novelle der F-Gas-Verordnung (EU, 2014) mit einem Phase-Down für die Produktion fluorierter Treibhausgase vorsieht.

Quoten eigenen sich für die Einführung einer neuen Technologie oder einer neuen Handlungsweise. Mit zunächst niedrigen und dann steigenden Quoten können sich die betroffenen Unternehmen langsam an neue Realitäten gewöhnen. Aber auch Quoten erfordern eine ständige Überprüfung ihrer Einhaltung und sind daher mit bürokratischem Aufwand verbunden. Beispiele für ein Quotenmodell ist die Beimischung von Bioethanol zu Ottokraftstoffen entsprechend der Erneuerbare-Energien-Richtlinie (EU, 2009).

Ein weiteres Steuerungsinstrument sind Haftungsregelungen. Dabei wird die Verursachung von Schäden mit empfindlichen Strafen belegt. Somit besteht der Anreiz, Schäden möglichst zu vermeiden. Auf welche Weise dies geschieht, bleibt dabei den Unternehmen ebenso freigestellt wie die Abwägung des Risikos. Haftungsregelungen können vor allem dann eingesetzt werden, wenn zu erwartende Schadensfälle eindeutig bestimmten Verursachenden zugewiesen werden können. Das ist bei Umweltschäden häufig nicht eindeutig oder nur unter großem Aufwand zu gewährleisten. Daher sind Haftungsregelungen im Kontext der Nachhaltigkeit wenig geeignet.

Auch wenn bereits Fortschritte hin zu einer nachhaltigeren Industrieproduktion zu verzeichnen sind, so ist der Status quo noch weit von wirklicher Nachhaltigkeit entfernt. So übersteigt etwa der Ressourcenverbrauch in Deutschland die Regenerationsfähigkeit der Erde um das Dreifache (o.V., 2020a). Auch die Stagnation der Entwicklung hin zu mehr Nachhaltigkeit in den letzten Jahren gibt großen Anlass zur Sorge. Mit einer bloßen Fortschreibung des gegenwärtigen Handelns lässt sich die Nachhaltigkeitstransformation der Industrie nicht im erforderlichen Maße voranbringen. Dabei sind bereits viele nachhaltige Technologien verfügbar. Allzu oft sind die ökonomischen Rahmenbedingungen Grund für das Scheitern.

Die Quintessenz: Es besteht dringender Bedarf an geeigneten Rahmenbedingungen, die den Strukturwandel weiter beschleunigen und nachhaltiges Handeln auch wirtschaftlich attraktiv machen. Bei der Gestaltung dieser Rahmenbedingungen ist darauf zu achten, dass auch im internationalen Handel faire Wettbewerbsbedingungen gelten, damit Leakage-Effekte vermieden werden. Nimmt die Implementierung nachhaltiger Technologien dann Fahrt auf, werden entsprechende Fachkräfte benötigt. 


\section{Handlungsempfehlungen}

Angesichts des voranschreitenden Klimawandels und der immer deutlicher werdenden katastrophalen Folgen der globalen Erwärmung besteht dringender Handlungsbedarf. Geeignete regulatorische Rahmenbedingungen sind die Voraussetzung für die Nachhaltigkeitstransformation der Industrie. Zunächst müssen langfristige, quantitative und spezifische Ziele vorgegeben werden. Für Treibhausgasemissionen sind im Klimaschutzplan 2050 bisher nur Sektorziele bis 2030 definiert. Für viele andere Nachhaltigkeitsfaktoren fehlen sektorspezifische Ziele. Darüber hinaus müssen Maßnahmen ergriffen werden, um nachhaltiges Handeln auch wirtschaftlich zu belohnen. Externalisierte Kosten für die Emission von $\mathrm{CO}_{2}$ und anderen Stoffen mit schädlicher Wirkung müssen dem Emissionsverursacher angelastet werden. Der nationale Brennstoffemissionshandel ist bei den angestrebten Preisen ein erster, aber nicht ausreichender Schritt. Für den Aufbau einer funktionierenden Kreislaufwirtschaft müssen produkt- und branchenspezifische Lösungen gefunden werden. Recyclingquoten und Sammelsysteme haben sich hier als wirksam erwiesen.

Um Wettbewerbsverzerrungen zu vermeiden, müssen auch für Importgüter Nachhaltigkeitsanforderungen gelten. Die Diskussion über die Erhebung einer $\mathrm{CO}_{2}$-Steuer auf Importgüter an den Grenzen des europäischen Wirtschaftsraums weist in die richtige Richtung. Die Umsetzung eines solchen Instruments ist indes hochkomplex und bedarf eines intensiven Austauschs mit den Betroffenen. Um schnelle Lernerfolge zu erzielen, sollten Instrumente wie Reallabore oder Wettbewerbe intensiver genutzt werden. Schnelle Erfolge sind insbesondere in der Flexibilisierung der Energieversorgung, der Elektrifizierung von Produktionsprozessen, dem Aufbau von Recyclingsystemen und der Entwicklung von Wasserstoff-, Kohlenwasserstoffsynthese- und Carbon-Capturing-Technologien erforderlich. Unternehmen sollten sich konstruktiv einbringen, Risiken eingehen und eine nachhaltige Zukunft mitgestalten. 


\section{Literatur}

Ahrens, Ralph H. (2019): Nützliche Bakterien, VDI Nachrichten. Online verfügbar unter: https://www.vdi-nachrichten.com/fokus/nuetzliche-bakterien/, zuletzt geprüft am 23.05.2020.

BMU (2019): Klimaschutz in Zahlen : Der Sektor Gebäude. Online verfügbar unter: https:// www.bmu.de/fileadmin/Daten_BMU/Download_PDF/Klimaschutz/klimaschutz_zahlen_2019_fs_industrie_de_bf.pdf, zuletzt geprüft am 23.05.2020.

Brunke, Jean Christian Ulf (2017): Energieeinsparpotenziale von energieintensiven Produktionsprozessen in Deutschland. University Stuttgart. Online verfügbar unter: https://elib.unistuttgart.de/bitstream/11682/9259/5/BRUNKE_ENERGIEEINSPARKOSTENKURVEN_209. pdf, zuletzt geprüft am 23.05.2020.

Bundesministerium für Wirtschaft und Energie (2020): Energiedaten, Bundesministerium für Wirtschaft und Energie. Online verfügbar unter: https://www.bmwi.de/Redaktion/DE/ Binaer/Energiedaten/energiedaten-gesamt-xls.xlsx?_blob=publicationFile\&v=121, zuletzt geprüft am 17.05.2020.

Diekmann, Jan et al. (2018): 'The LithoRec Process', in Sustainable Production, Life Cycle Engineering and Management. https://doi.org/10.1007/978-3-319-70572-9_2.

European Commission (2015): EU ETS Handbook, Climate Action. Online verfügbar unter: http://ec.europa.eu/clima/publications/docs/ets_handbook_en.pdf, zuletzt geprüft am 17.05.2020.

Europäische Union (2009): Richtlinie 2009/28/EG des Europäischen Parlaments und des Rates vom 23. April 2009 zur Förderung der Nutzung von Energie aus erneuerbaren Quellen und zur Änderung und anschließenden Aufhebung der Richtlinien 2001/77/EG und 2003/30/EG. Online verfügbar: https://eur-lex.europa.eu/legal-content/DE/TXT/?uri=CELEX\%3A32009L0028, zuletzt geprüft am 21.09.2020.

Europäische Union (2014): VERORDNUNG (EU) Nr. 517/2014 DES EUROPÄISCHEN PARLAMENTS UND DES RATES vom 16. April 2014 über fluorierte Treibhausgase und zur Aufhebung der Verordnung (EG) Nr. 842/2006. Online verfügbar: https://eur-lex.europa. eu/legal-content/EN/TXT/?uri=CELEX\%3A32014R0517, zuletzt geprüft am 21.09.2020.

eurostat (2019): Energy statistics - an overview, eurostat. Online verfügbar unter: https:// ec.europa.eu/eurostat/statistics-explained/index.php/Energy_statistics_-_an_overview\#Final_energy_consumption, zuletzt geprüft am 17.05.2020.

Harloff, Thomas (2019): Garantien bei Elektrofahrzeugen. Online verfügbar unter: https:// www.auto-motor-und-sport.de/tech-zukunft/alternative-antriebe/diese-garantien-gewaehren-hersteller-bei-e-auto-akkus/, zuletzt geprüft am 23.05.2020.

Hausfather, Zeke (2018): 'Analysis: How much "carbon budget" is left to limit global warming to $1.5 \mathrm{C}$ ?', Carbon Brief.

Intergovernmental Panel on Climate Change (2014): Climate Change 2014 Mitigation of Climate Change, Climate Change 2014 Mitigation of Climate Change. https://doi. org/10.1017/cbo9781107415416. 
Matteini, Marco, Pasqualetto, Giorgia and Petrovska, Ana (2018): 'Cost-benefit analysis of energy management systems implementation at enterprise and programme level', in Eceee Industrial Summer Study Proceedings.

Michaelis, Dr. Sarah et al. (2018): Roadmap Batterieproduktionsmittel 2030. Frankfurt am Main. Online verfügbar unter: http://battprod.vdma.org, zuletzt geprüft am 17.05.2020.

Müller, Jochen (2017): BMW Group demonstriert Führungsrolle im Bereich Elektromobilität. Leipzig. Online verfügbar unter: https://www.bmwgroup.com/content/dam/grpw/websites/bmwgroup_com/responsibility/downloads/de/2018/171025-BMW-Group-Fuehrungsrolle-Elektromobilitaet.pdf, zuletzt geprüft am 17.05.2020.

Neligan, Adriana; and Schmitz, Edgar (2017): Digitale Strategien für mehr Materialeffizienz in der Industrie: Ergebnisse aus dem IW-Zukunftspanel. Online verfügbar unter: https:// www.econstor.eu/bitstream/10419/157205/1/IW-Report-2017-03.pdf, zuletzt geprüft am 17.05.2020.

o. V. (2019a): No Title. Available at: https://www.solpart-project.eu, zuletzt geprüft am 17.05.2020.

o. V. (2019b): Sunfire entwickelt effizientere Hochtemperatur-Elektrolyse. Online verfügbar unter: https://www.chemietechnik.de/sunfire-entwickelt-effizientere-hochtemperaturelektrolyse/, zuletzt geprüft am 23.05.2020.

o. V. (2020a): Country Overshoot Days. Online verfügbar unter: https://www.overshootday. org/newsroom/country-overshoot-days/, zuletzt geprüft am 23.05.2020.

o. V. (2020b): Rohstoffe in deutschen Schubladenhandys. Online verfügbar unter: https:// www.informationszentrum-mobilfunk.de/mediathek/grafiken-bilder/rohstoffe-in-deutschen-schubladenhandys, zuletzt geprüft am 23.05.2020.

Philp, Jim C., Ritchie, Rachael J. and Allan, Jacqueline E. M. (2013): 'Biobased chemicals: The convergence of green chemistry with industrial biotechnology', Trends in Biotechnology. https://doi.org/10.1016/j.tibtech.2012.12.007.

Prengaman, R. David; Mirza, A. H. (2017): 'Recycling concepts for lead-acid batteries', in Lead-Acid Batteries for Future Automobiles. https://doi.org/10.1016/B978-0-444-637000.00020-9.

Rudschies, Wolfgang (2019): Elektroauto-Akkus: So funktioniert das Recycling. Online verfügbar unter: https://www.adac.de/rund-ums-fahrzeug/elektromobilitaet/info/elektroautoakku-recycling/, zuletzt geprüft am 23.05.2020.

Stagge, Mark (2019): Pressemitteilung. Essen. Online verfügbar unter: https://www.thyssenkrupp-steel.com/media/content_1/unternehmen_3/nachhaltigkeit/hydrogen2steel/wasserstoff_phase_1.pdf, zuletzt geprüft am 17.05.2020.

Umweltbundesamt (2019a): Der Europäische Emissionshandel, Umweltbundesamt. Online verfügbar unter: https://www.umweltbundesamt.de/daten/klima/der-europaeische-emissionshandel, zuletzt geprüft am 23.05.2020.

Umweltbundesamt (2019b): Emission von Feinstaub der Partikelgröße PM-10, Umweltbundesamt. Online verfügbar unter: https://www.umweltbundesamt.de/daten/luft/luftschad- 
stoff-emissionen-in-deutschland/emission-von-feinstaub-der-partikelgroesse-pm10, zuletzt geprüft am 23.05.2020.

Umweltbundesamt (2020): Energieverbrauch nach Energieträgern und Sektoren, Umweltbundesamt. Online verfügbar unter: https://www.umweltbundesamt.de/daten/energie/ energieverbrauch-nach-energietraegern-sektoren, zuletzt geprüft am 18.05.2020.

Weinhold, Nicole (2019): Versuchsanlage aus Bioreaktor und Elektrolyseur, Erneuerbare Energien. Online verfügbar unter: https://www.erneuerbareenergien.de/versuchsanlageaus-bioreaktor-und-elektrolyseur, zuletzt geprüft am 23.05.2020.

Zinke, Guido (2019): Anwendung künstlicher Intelligenz im Energiesektor. Online verfügbar unter: https://vdivde-it.de/de/anwendung-kuenstlicher-intelligenz-im-energiesektor, zuletzt geprüft am 23.05.2020.

\section{(c) (1)}

Dieses Kapitel wird unter der Creative Commons Namensnennung 4.0 International Lizenz http://creativecommons.org/licenses/by/4.0/deed.de) veröffentlicht, welche die Nutzung, Vervielfältigung, Bearbeitung, Verbreitung und Wiedergabe in jeglichem Medium und Format erlaubt, sofern Sie den/die ursprünglichen Autor(en) und die Quelle ordnungsgemäß nennen, einen Link zur Creative Commons Lizenz beifügen und angeben, ob Änderungen vorgenommen wurden.

Die in diesem Kapitel enthaltenen Bilder und sonstiges Drittmaterial unterliegen ebenfalls der genannten Creative Commons Lizenz, sofern sich aus der Abbildungslegende nichts anderes ergibt. Sofern das betreffende Material nicht unter der genannten Creative Commons Lizenz steht und die betreffende Handlung nicht nach gesetzlichen Vorschriften erlaubt ist, ist für die oben aufgeführten Weiterverwendungen des Materials die Einwilligung des jeweiligen Rechteinhabers einzuholen. 